\title{
Nuevos hallazgos de los moluscos Nemrac carmenensis Clark 1946 (Gastropoda, Turbinoidea, Turbinidae) y Solena (Eosolen) bolivarensis Clark 1946 (Bivalvia - Solenida - Solenidae): Precisiones estratigráficas y paleoambientales para las faunas del Eoceno tardío del Caribe colombiano
}

\author{
Hermann Darío Bermúdez ${ }^{\mathrm{a}, *}$ \\ ${ }^{a}$ Grupo de Investigación Paleoexplorer. Calle 98BISNo. 71D-20, Bogotá D.C., Colombia. \\ *hermann.bermudez@paleoexplorer.net
}

\begin{abstract}
Resumen
Se reporta el hallazgo de nuevos ejemplares del gasterópodo Nemrac carmenensis Clark 1946 y del bivalvo Solena (Eosolen) bolivarensis Clark 1946, en rocas del Paleógeno del Cinturón Plegado de San Jacinto, Caribe colombiano; complementando la información disponible de estas especies, de las cuales solo se habían colectado ejemplares únicos durante la primera mitad del siglo pasado. Este descubrimiento permite establecer la posición estratigráfica de estas y otras faunas halladas previamente en el Eoceno del Caribe colombiano en la parte alta de la Formación Arroyo Seco, asignándole una edad Bartoniense - Priaboniense (Eoceno tardío) y un paleoambiente de depósito de frente deltáico.
\end{abstract}

Palabras clave: Caribe, Cinturón Plegado de San Jacinto, Colombia, Eoceno tardío, Formación Arroyo Seco, moluscos.

\begin{abstract}
New specimens of the gastropod Nemrac carmenensis Clark 1946 and the bivalve Solena (Eosolen) bolivarensis Clark 1946, in Paleogene rocks of San Jacinto Fold Belt (SJFB), Colombian Caribbean region were found and reported to complement the information available for these species. These findings of unique specimens had only been collected during the first half of the last century. This discovery allows to define the stratigraphic position of these and other faunas previously found in the Eocene of the Colombian Caribbean region at the upper levels of Arroyo Seco Formation, allowing the assignment of a Late Eocene age (Bartonian - Priabonian) and a delta-front environment.
\end{abstract}

Palabras clave: Caribbean, San Jacinto Fold Belt, Colombia, late Eocene, Arroyo Seco Formation, mollusks.

\section{Introducción}

El gasterópodo Nemrac carmenensis Clark 1946 y el bivalvo Solena (Eosolen) bolivarensis Clark 1946, fueron descritos por el Dr. Bruce L. Clark (Clark y Durham, 1946) a partir de ejemplares únicos colectados en trabajos de campo de la Standard Oil Company y del paleontólogo estadounidense Dr. Frank Marion Anderson (Anderson, 1928), en la región de Carmen de Bolívar, Colombia, durante la primera mitad del siglo XX. Los fósiles aquí estudiados forman parte de una serie de moluscos paleógenos, poco conocidos (la mayoría endémicos), que incluyen numerosas especies de bivalvos y gasterópodos, así como algunos registros de nautiloideos, corales y crustáceos, de los cuales no existían nuevos reportes desde hace ya casi 70 años.

Los trabajos originales de Anderson (1928) y Clark y Durham (1946) no presentaban una localización geográfica ni estratigráfica precisa, por lo que la posición exacta de estos hallazgos era prácticamente desconocida, lo que desafortunadamente restó valor a la importancia de sus estudios, claves para la paleontología del Caribe colombiano y del norte de Sur América. Teniendo en 
cuenta esto, se realizó una búsqueda de las localidades fosilíferas reportadas de manera aproximada por ellos y tras su hallazgo, se realizó la descripción estratigráfica y la colecta de abundantes ejemplares de moluscos, cuyo estudio aún se encuentra en desarrollo, pero arroja ya sus primeros resultados.

El presente trabajo reporta el descubrimiento de nuevos ejemplares de moluscos en el área de los hallazgos originales, lo que ha permitido ubicar de forma precisa, estratigráfica y geográficamente, estos horizontes fosilíferos y en particular, colectar nuevos ejemplares de especies de los que solo existía el holotipo; así como describirlos e ilustrarlos sin las limitaciones editoriales de mediados del siglo pasado. La descripción detallada de las secuencias sedimentarias aflorantes, ha permitido además conocer la litología, facies y paleoambientes sedimentarios de estos horizontes. Determinaciones micropaleontológicas recientes aportan la edad precisa de estos depósitos.

La fauna de moluscos del Eoceno reportada en los trabajos del siglo pasado guarda información valiosa acerca de la evolución, paleoambientes y paleogeografía del norte de Sur América durante el Paleógeno. El redescubrimiento de estos fósiles y su integración dentro del escenario estratigráfico y evolutivo del Caribe colombiano, permite tener un marco de referencia para los nuevos hallazgos que se están realizando en el área y brindan una mejor comprensión de la importancia de los estudios paleontológicos de Anderson (1928) y Clark y Durham (1946), pioneros en el estudio de los moluscos paleógenos de Colombia; así como de los nuevos datos por descubrir, permitiendo además, por primera vez, tener ejemplares de esta malacofauna en las colecciones paleontológicas de Colombia para su estudio por parte de los investigadores nacionales.

El material estudiado está depositado en la Colección Paleontológica del Departamento de Geociencias de la Universidad Nacional de Colombia, sede Bogotá, con el acrónimo UN-DG. El criterio de clasificación que se sigue en los gasterópodos es el de Bouchet et al., 2005 y en los bivalvos el del Treatise on Invertebrate Paleontology (Moore y Teichert, 1969) y su terminología revisada recientemente (Carter et al., 2012).

\section{Localización y marco geológico}

El horizonte fosilífero que contiene los ejemplares aquí descritos se localiza sobre el arroyo Mancomoján $\left(75^{\circ} 16^{\prime} 12^{\prime \prime} \mathrm{W}\right.$; 9037'28' $\left.\mathrm{N}\right)$, a unos $2.3 \mathrm{~km}$ al NW del municipio de Pijiguay, departamento de Bolívar, Colombia (Figura 1). Geológicamente el nivel muestreado se ubica en el flanco oeste de anticlinal El Cedro, sobre la parte alta de la Formación Arroyo Seco y corresponde a una litoarenita fosilífera de grano fino, de color gris verdoso, con cemento calcáreo y abundantes restos de moluscos, fragmentos leñosos y de carbón (Figura 2).
De acuerdo a los datos suministrados por Clark y Durham (1946), este nivel correspondería aproximadamente o estaría muy cerca de los niveles fosilíferos descritos en su localidad S-60, así como dentro de la misma estructura y unidad litoestratigráfica de otras localidades, que ellos ubicaron en la denominada Zona C (ver Figura 1).

Los moluscos aquí descritos, a pesar de haber sido encontrados en un mismo horizonte fosilífero, habían sido reportados originalmente por Clark y Durham (1946) en zonas diferentes y exclusivas; Nemrac carmenensis fue reportado en la localidad CAS 31694 (Zona B), mientras que Solena (Eosolen) bolivarensis fue reportado en la localidad S-60 (Zona C), lo que pone en duda la utilidad de dichas zonas, lo cual debe revisarse en el futuro.

\section{Estratigrafía}

La Formación Arroyo Seco (Paleoceno tardío - Eoceno tardío), constituye la base del Paleógeno del Cinturón Plegado de San Jacinto (CPSJ) y está constituida por proporciones variables de conglomerados polimícticos (particularmente importantes hacia la base de la unidad), arenitas líticas y lodolitas. La unidad reposa discordantemente sobre la Formación Cansona (Coniaciano - Maastrichtiano) y es a su vez suprayacida por las calizas de la Formación Toluviejo o las areniscas fosilíferas de la Formación San Jacinto (Eoceno tardío - Oligoceno), las cuales representan facies heterópicas isócronas que se interdigitan en esta región del CPSJ (Bermúdez et al., 2009).

La edad de la Formación Arroyo Seco ha sido determinada con foraminíferos, palinología y nanofósiles calcáreos, como Paleoceno medio - Eoceno tardío (Herrera et al., 2009; Universidad de Caldas - ANH, 2009; Bermúdez et al., 2009) e incluye además diversos horizontes fosilíferos con moluscos marinos y de agua salobre-dulce (Bermúdez et al., 2013). Los foraminíferos planctónicos ilustran la presencia de las zonas P3 a P16 de Berggren y Pearson (2005); a partir de nanoplancton calcáreo se identifican las zonas NP5 a NP20 de Martini (1971), mientras que los palinomorfos muestran la presencia de las zonas T02-T07 de Jaramillo et al., (2009).

Las determinaciones bioestratigráficas disponibles para la parte alta de la unidad (en donde se encuentran los niveles fosilíferos de moluscos de afinidad marina aquí descritos), obtenidas de estudios de pozos y secciones aledañas (ATG-ANH, 2009; Universidad de Caldas ANH, 2009), restringen la edad de depósito del techo de la Formación Arroyo Seco al Bartoniense - Priaboniense (Eoceno tardío 40.4 - 33.9 m.a), especialmente con base en la presencia de foraminíferos como Acarinina collactea, A. bullbrooki, A. medizzai, A. primitiva, A. rohri, A. topilensis, Clavigerinella colombiana, C. eocanica, Globigerina officinalis, G. linaperta, Igorina broedermanni, Morozovella spinulosa, Paragloborotalia griffinoides, $P$. nana, Planorotalites pseudoscitula, Subbotina eocaena, 


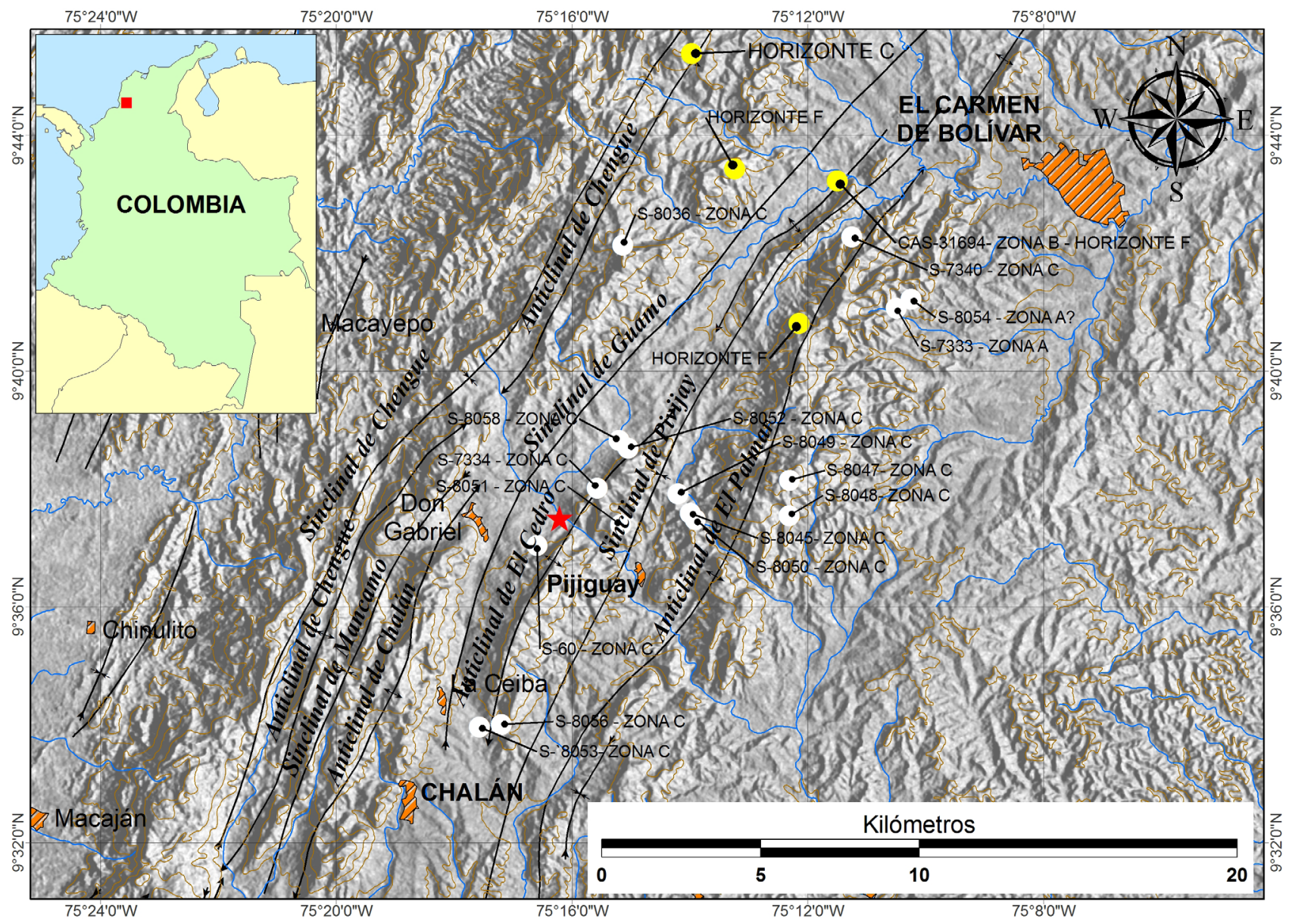

Figura 1. Mapa de localización del horizonte fosilífero descrito en este trabajo (estrella roja), al NW del municipio de Pijiguay, Departamento de Bolívar, Colombia, ilustrando además la posición aproximada de las localidades de Clark y Durham (1946) — puntos blancos—y de Anderson (1928) —puntos amarillos-.

S. linaperta, Turborotalia pomeroli, T. cerroazulensis y T. posagnoensis y los nanofósiles calcáreos Chiasmolithus solitus, C. nitidus, Helicosphaera lophota, H. seminulum, Nannotetrina cristata, Reticulofenestra bisecta, R. reticulata y Sphenolithus radians.

\section{Paleontología sistemática}

Clase Gastropoda Cuvier, 1797 Superfamilia Turbinoidea Rafinesque, 1815

Familia Turbinidae Rafinesque, 1815

Género Nemrac Clark, 1946

Tipo. Nemrac carmenensis Clark, 1946, Eoceno, región Caribe de Colombia.

Nemrac carmenensis Clark, 1946

(Figura 3)
Nemrac carmenensis Clark, 1946, p. 14, lám. 19, fig. 8, 14, 15 .

Descripción. Los tres hipotipos descritos presentan una concha pequeña turbinada, relativamente alta (casi $3 / 4$ del ancho máximo) con $5 \frac{1}{2}$ vueltas, de las cuales $1 \frac{1}{2}$ a 2 corresponden a la protoconcha, la cual es baja, casi planispiral y ligeramente inclinada y luce como una continuación del resto de la concha. Las vueltas de la teleoconcha son lisas, suavemente convexas y presentan en su parte interna una banda sutural crenulada estrecha, definida por un surco granulado, que desaparece en la protoconcha, lo que la diferencia de la teleoconcha (Figura $3 \mathrm{D})$; la base de la concha, muy bien definida en el hipotipo UN-DG-GT-005a (Figura 3 B), presenta abertura circular y ombligo abierto del que irradian costillas irregulares separadas por un surco.

Material examinado. Tres especímenes (en excelente condición), identificados con los números de catálogo UN-DG-GT-005a, UN-DG-GT-005b, y UN-DG-GT-005c. 


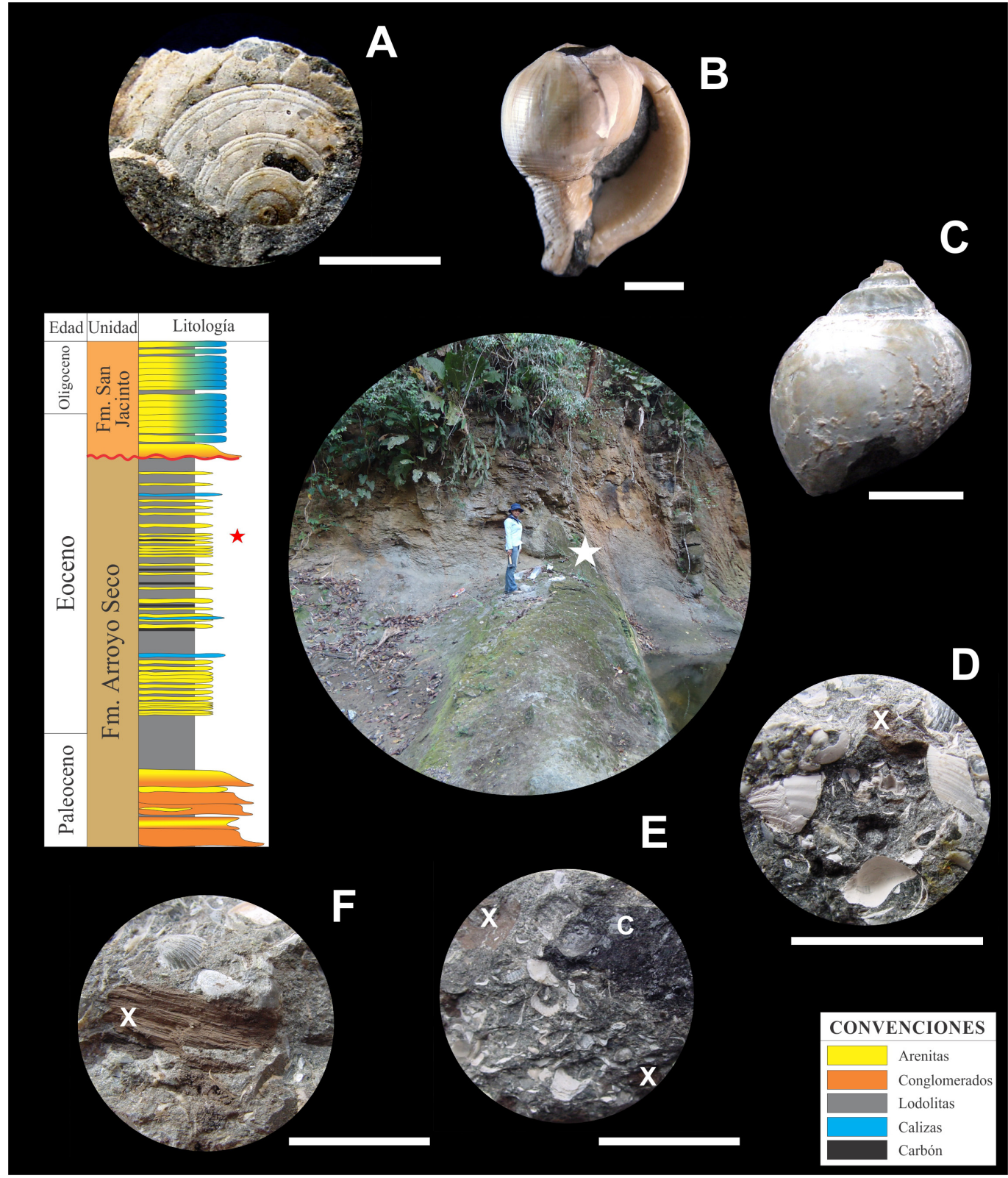

Figura 2. Columna estratigráfica, aspecto del afloramiento, detalle de las facies y alguna de la fauna asociada de moluscos. A) Architectonica sp. B) Peruficus sp. C) Crommium sp. D-F) Aspecto de la facies de litoarenitas fosilíferas con fragmentos de carbón (c) y restos leñosos (x) donde se encontraron los moluscos aquí descritos. La barra equivale a $1 \mathrm{~cm}$.

El holotipo (a la vez genotipo) de Nemrac carmenensis, está depositado en el Departamento de Paleontología de la Academia de Ciencias de California, CAS, por sus siglas en inglés, (Localidad CAS 31694, espécimen No. 7936) y no pudo ser consultado.

\section{Dimensiones.}

Holotipo CAS 31694, No. 7936: Altura= $4.1 \mathrm{~mm}$; ancho máximo $=5.7 \mathrm{~mm}$.

Hipotipo UN-DG-GT-005a: Altura $=4.3 \mathrm{~mm}$; ancho máximo $=6.5 \mathrm{~mm}$.

Hipotipo UN-DG-GT-005b: Altura $=4.2 \mathrm{~mm}$; ancho máximo $=6.1 \mathrm{~mm}$.
Hipotipo UN-DG-GT-005c: Altura $=5.0 \mathrm{~mm}$; ancho máximo $=6.6 \mathrm{~mm}$.

Ocurrencia. Pijiguay, Carmen de Bolívar. Región Caribe colombiana.

Discusión. Los tres ejemplares encontrados corresponden inequívocamente a la descripción original del holotipo de Clark (1946), quien de acuerdo a sus características únicas erigió una nueva especie (así como un nuevo género), que hasta la fecha solo se ha reportado en el Eoceno del Caribe colombiano. Originalmente el género Nemrac fue asignado a la familia Turbinidae aunque posteriormente Sepkoski (2002) lo asignó a la orden Archaeogastropoda. Sin tener 


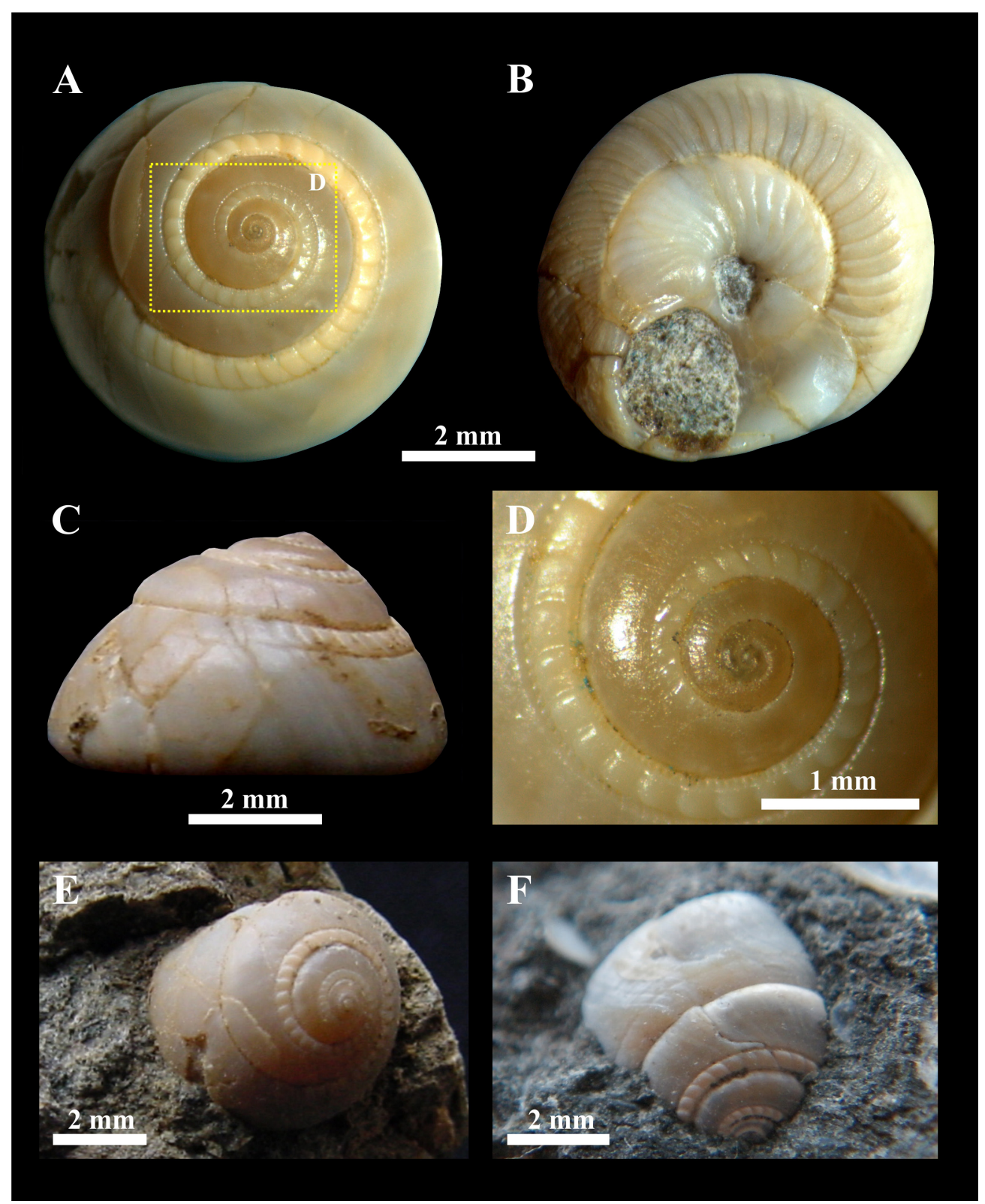

Figura 3. Nemrac carmenensis Clark 1946. Formación Arroyo Seco, Colombia. A-D) Hipotipo UN-DG-GT-005a. A) Vista superior B) Vista basal. C) Vista lateral. D) Detalle de la protoconcha. E) Hipotipo UN-DG-GT-005b. F) Hipotipo UN-DG-GT-005c.

acceso al holotipo y solo con la descripción de los tres ejemplares hallados en este trabajo, se prefiere mantener la asignación original de Clark (1946).

Las dimensiones de los ejemplares colectados en este trabajo son muy similares entre sí, aunque ligeramente más grandes a las del holotipo, lo que permite suponer que la especie tiene un tamaño cercano a los $6 \mathrm{~mm}$.

Clase: Bivalvia Linnaei, 1758

Subclase: Autobranchia Groblen, 1894

Infraclase: Heteroconchia Cox, 1960

Orden: Solenida Dall, 1889

Superfamilia: Solenoidea Lamarck, 1809

Familia: Solenidae Lamarck, 1809
Género: Solena Mörch, 1853

Tipo. Solen obliquus Spengler. Reciente. Caribe.

Subgénero: Eosolen Stewart, 1930

Tipo. Solen plagiaulax Cossmann. Eoceno. Cuenca de París.

\section{Solena (Eosolen) bolivarensis Clark, 1946}

(Figura 4)

Solena (Eosolen) bolivarensis Clark, 1946, p. 73, lám. 13, fig. 17, 18.

Descripción. Concha alargada, relativamente gruesa, 
con bordes dorsal y ventral paralelos y aproximadamente rectos; extremo anterior redondeado (solo se preserva éste en el hipotipo UN-DG-BV-010, Figura 4 A); superficie lisa con notorias líneas de crecimiento.

Material examinado. Un espécimen con buena preservación (solamente se conservó la valva derecha), identificado con el número de catálogo UN-DG-BV-010.

El holotipo de Solena (Eosolen) bolivarensis, está depositado en el Museo de Paleontología de la Universidad de California, Berkeley, UC por sus siglas en inglés (Localidad UC S60, espécimen No. 34915) y no pudo ser consultado.

\section{Dimensiones.}

Holotipo UC S60 No. 34915: Altura= $21.5 \mathrm{~mm}$; ancho máximo del ejemplar roto $=46 \mathrm{~mm}$.

Hipotipo UN-DG-BV-010: Altura= $20 \mathrm{~mm}$; ancho máximo del ejemplar roto $=61 \mathrm{~mm}$.

Ocurrencia. Pijiguay, departamento de Bolívar. Región Caribe colombiana.

Discusión. A pesar de contar con un solo ejemplar, las características morfológicas coinciden claramente con la descripción original de Solena (Eosolen) bolivarensis de Clark, 1946, la cual constituye una forma endémica solo descrita en el Eoceno de Colombia. Al igual que en la descripción original, donde la especie fue referida con reservas al subgénero Eosolen, debido a la ausencia del surco umbonal tan común en la mayoría de especies de Eosolen, la preservación del nuevo ejemplar (solo una valva) no permite una asignación exenta de dudas. De cualquier manera se coincide con el autor original en que las líneas irradiantes en el borde anterior dorsal sugieren una posible relación con Eosolen.

A partir de las proporciones observadas en los dos ejemplares existentes, se calcula un ancho máximo aproximado de $85 \mathrm{~mm}$.

\section{Precisiones estratigráficas y paleoambientales}

El hallazgo de estos dos ejemplares (de distintas zonas) en un mismo horizonte fosilífero, sugiere que las tres zonas definidas por Clark y Durham (1946) no necesariamente corresponden a niveles con faunas exclusivas y que la posición estratigráfica relativa de estos dos moluscos es incierta. La presencia del gasterópodo Faunus (Pseudofaunus) cf. chiraensis en la "Zona A" de dichos autores, refuerza esta idea, ya que Faunus es un gasterópodo de aguas salobres y dulces, que actualmente vive en la desembocadura de los ríos en el Pacífico occidental (Woodring, 1973), y es muy similar a los gasterópodos encontrados en la Formación

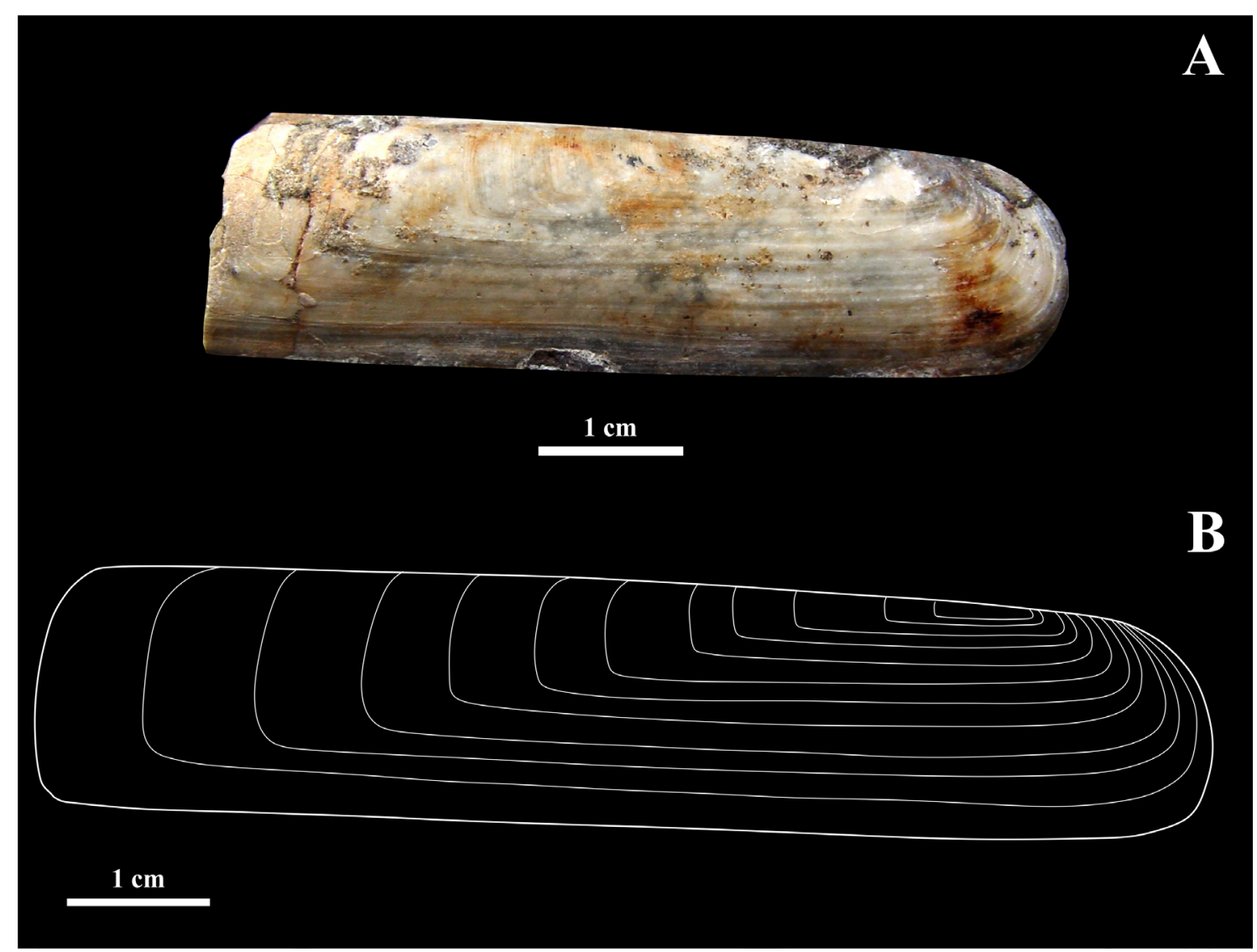

Figura 4. Solena (Eosolen) bolivarensis Clark 1946. A) Aspecto del hipotipo UN-DG-BV-010. B) Reconstrucción esquemática a partir de la información suministrada por el holotipo y el hipotipo hallado en este trabajo. 
Arroyo Seco en el área de Chalán (unos $10 \mathrm{~km}$ al sureste de la localidad aquí estudiada), que incluyeron además de Faunus sp., Pachychilus canoasensis, Hannatoma gesteri y H. emendorferi (Bermúdez et al., 2013), en niveles carbonosos de clara afinidad paleoambiental con zonas pantanosas; lo que sugiere que proviene de un horizonte diferente, quizás más inferior. Esta situación es clara en el área de Chalán, donde los horizontes con fósiles marinos solo se desarrollan en la parte alta de la unidad; mientras que los horizontes carbonosos con fósiles se localizan sobre la parte media de la Formación Arroyo Seco, tal como también se pudo apreciar en el arroyo Mancomojan, hacia el núcleo del anticlinal El Cedro, unos 100 m estratigráficos por debajo del horizonte aquí descrito.

De cualquier manera el conjunto de la mayoría de hallazgos fósiles del CPSJ conforman una serie de asociaciones faunísticas muy bien definidas, que reflejan condiciones de depósito muy someras y clima tropical, que se asocia a depósitos marinos de litoral, sometidos al influjo de las olas y las tormentas y de frente deltaico (Anderson, 1928; Clark y Durham, 1946; ATG-ANH, 2008; Universidad de Caldas - ANH, 2009; Bermúdez et al., 2009), que no sustentan la idea de que el Paleógeno del CPSJ se acumuló en paleoambientes marinos profundos, incluyendo turbiditas y depósitos de zonas de talud, batiales y abisales, tal como plantean muchos estudios (Duque-Caro, 1972, Duque-Caro et al.,1996; Caro, 2003; Guzmán et al., 2004, entre otros).

El abundante registro fósil del Eoceno del Caribe colombiano, es afín al encontrado en las asociaciones del Eoceno y Oligoceno del Perú (Olsson, 1929, 1930, 1931; Woods, 1922), Centro América e incluso las Antillas y EEUU (Maury, 1912; Aldrich, 1921; Clark y Durham, 1946; Woodring, 1973), aunque muchas de las formas descritas parecen ser endémicas (Clark y Durham, 1946).

Estudios en desarrollo están empezando a redescubrir la paleontología de moluscos del Caribe colombiano, con la colecta y estudio de nuevo material, lo que unido a la descripción estratigráfica detallada, la micropaleontología y la cartografía regional será clave en el entendimiento de las relaciones faunísticas de Norte y Sur América

\section{Agradecimientos}

El presente estudio fue financiado por el Grupo de Investigación Paleoexplorer, como parte del proyecto "Moluscos del Caribe colombiano". Se agradece la colaboración de Liliana Bolívar en la elaboración del material gráfico y de Luz Stella Bolívar en la fase de campo. El autor agradece de forma especial a Francisco J. Vega y un revisor anónimo, así como al editor en jefe Dr. Josep Moreno-Bedmar, por sus valiosos aportes y sugerencias para mejorar el manuscrito original.

\section{References}

Aldrich, T.H., 1921, New Eocene species from Alabama: Bulletins of American Paleontology, 9 (37), 1-32.

Anderson, F.M., 1928, Notes on Lower Tertiary deposits of Colombia and their molluscan and foraminiferal fauna: Proceedings of the California Academy of Sciences, Fourth Series, 17 (1), 1-29.

Asesorías Técnicas Geológicas - Agencia Nacional de Hidrocarburos (ATGANH), 2009, Cartografía geológica, levantamiento de columnas estratigráficas, toma de muestras y análisis bioestratigráficos. Sector de Chalán (Cuenca Sinú-San Jacinto): Reporte interno a la ANH con 12 anexos, disponible en <http://www.anh.gov.co/es/index. php?id=133>, consultado: 20 de junio de 2011.

Berggren, W.A., Pearson, P.N., 2005, A revised tropical and subtropical Paleogene planktonic foraminiferal zonation: Journal of Foraminiferal Research, 35 (4), 279-298.

Bermúdez, H.D., Alvarán, M., Grajales, J.A., Restrepo, L.C., Rosero, J.S., Guzmán, C., Ruiz, E.C., Navarrete, R.E., Jaramillo, C., Osorno, J.F., 2009, Estratigrafía y evolución geológica de la secuencia sedimentaria del Cinturón Plegado de San Jacinto, en Memorias XII, congreso colombiano de Geología: Paipa, 100-123.

Bermúdez, H.D., Vega, F.J., Corredor, V.E., Numpaque, A., 2013, Los moluscos del Paleógeno del CPSJ y su relevancia en las interpretaciones paleoambientales del Caribe colombiano (resumen), en Memorias XIV Congreso Colombiano de Geología: Paipa, 333.

Bouchet, P., Rocroi, J.P., Fryda, J., Hausdorf, B., Ponder, W., Valdés, Á., Warén, A., 2005, Classification and Nomenclator of Gastropod Families: Malacologia, 47 (1/2), 1-397.

Caro, M., 2003, Structural evolution of the San Jacinto fold belt, NW Colombia: Calgary, Alberta, Canada, University of Calgary, thesis of Master of Science, $82 \mathrm{p}$.

Carter, J.G., Harries, P.J., Malchus, N., Sartori, A.F., Anderson, L.C., Bieler, R., Bogan, A.E., Coan, E.V., Cope, J.C.W., Cragg, S., Garcia-March, J., Hylleberg, J., Kelley, P., Kleemann, K., Kriz, J., McRoberts, C., Mikkelsen, P.M., Pojeta, J., Skelton, P.W., Tëmkin, I., Yancey, T., Zieritz A., 2012, Part N, Revised, Volume 1, Chapter 31: Illustrated Glossary of the Bivalvia. Treatise Online, 48, 1-209.

Clark, B.L., Durham, J.W., 1946, Eocene Faunas from the Department of Bolívar, Colombia: Geological Society of America, Memoir 16, New York, $126 \mathrm{p}$.

Cox, L.R., 1960, Thoughts on the classification of the Bivalvia: Proceedings of the Malacological Society of London, 34, 60-88.

Cuvier, G., 1797, Tableau élémentaire de l'histoirenaturelle des animaux: Paris, Baudouin, 710 p.

Dall, W.H., 1889, On the hinge of pelecypods and its development with an attempt toward a better subdivision of the group: American Journal of Science, series 3, 38, 445-462.

Duque-Caro, H., 1972, Ciclos tectónicos y sedimentarios en el Norte de Colombia y sus relaciones con la Paleoecología: Boletín Geológico Instituto Nacional de Investigaciones Geológico Mineras, Bogotá, 19 (3), 1-23.

Duque-Caro, H., Guzmán, G., Hernández, R., 1996, Mapa geológico de la plancha 38, Carmen de Bolívar, escala 1:100000: Bogotá, Colombia, Ingeominas, 1 mapa con texto.

Groblen, C., 1894, Zur Kenntnis der Morphologie, der Verwandtschaftsverhältnisse und des Systems der Mollusken: Sitzungsberichte der Mathematisch-Naturwissenschaftlichen Klasse der Kaiserlichen Akademie der Wissenschaften, 103 (1), 61-86.

Guzmán, G., Gómez, E., Serrano, B., 2004, Geología de los Cinturones del Sinú, San Jacinto y borde occidental del Valle Inferior del Magdalena, Caribe Colombiano, escala 1:300000: Memoria técnica Ingeominas. Bogotá, 134 p.

Herrera, J., Bermúdez, H., Alfonso, M., Calderón, J., Pardo, A., Lozano, A., 2009, Cartografía Geológica de un área del Cinturón Plegado de San Jacinto, en Memorias $10^{\text {th }}$ Simposio Bolivariano Exploración Petrolera en Cuencas Subandinas, Cartagena, Colombia, 1-10. 
Jaramillo, C., Rueda,M., Bayona, G., Santos, C., Flórez, P., Parra, F., 2009 Biostratigraphy breaking paradigms: Dating the Mirador Formation in the Llanos Basin of Colombia, en Demchuck, T. (ed), Geologic problems solving with microfossils: A volume in honor of Gary D. Jones, Society for Sedimentary geology, Special Publication 93, Tulsa, 29-40.

Lamarck, J.B.P. de, 1809, Philosophie zoologique: Dentu, Paris, 1, 428 p

Linnaei, C., 1758, Systema Naturae per Regna Tria Naturae, secundum Classes, Ordines, Genera, species, cum Characteribus, Differentiis, Synonymis, locis: Tomus I, EditioDecima, Reformata, Holmiae, 824 p.

Martini, E., 1971, Standard Tertiary and Quaternary Calcareous Nannoplankton Zonation, en Farinaci, A. (ed), Proceeding of the $2^{\text {nd }}$ Planktonic Conference, Roma, Edizioni Tectonscienzas, 2, 739-785.

Maury, C.J., 1912, A contribution to the paleontology of Trinidad: Journal of the Academy of Natural Sciences of Philadelphia, 15, 1-112.

Moore, R.C., Teichert, C., (eds.), 1969, Treatise on Invertebrate Paleontology: Lawrence, Kansas, University of Kansas Press, Part N, Mollusca 6: Bivalvia, 1-2, xxxvii +952 p.

Mörch, O.A.L., 1853, Catalogus Conchyliorumquae Reliquit D. Alphonso d'Aguirra \& Gadea, comes de Yoldi. Fasc. Sec.: Acephala. Hafniae, $74 \mathrm{p}$.

Olsson, A.A., 1929, Contributions to the Tertiary paleontology of northern Peru: Part 2. Upper Eocene Mollusca and Brachiopoda: Bulletins of American Paleontology, 15 (57), 1-96.

Olsson, A.A., 1930, Contributions to the Tertiary Paleontology of Northern Peru: Part 3. Eocene Mollusca: Bulletins of American Paleontology, 17 (62), 1-96.
Olsson, A.A., 1931, Contributions to the Tertiary Paleontology of Northern Peru: Part 4. The Peruvian Oligocene: Bulletins of American Paleontology, 17 (63), 97-264.

Rafinesque, C.S., 1815, Analyse de la nature, ou Tableau de l'univers et des corps organisées: Palermo, $224 \mathrm{p}$.

Sepkoski, J.J., 2002, A compendium of fossil marine animal genera: Bulletins of American Paleontology, 363, 1-560.

Stewart, R.B., 1930, Gabb's California Cretaceous and Tertiary Type Lamellibranchs: The Academy of Natural Sciences of Philadelphia, Special Publication, 3, 1-314.

Universidad de Caldas - Agencia Nacional de Hidrocarburos (ANH), 2009, Estudio integrado de los núcleos y registros obtenidos de los pozos someros tipo "slim holes" en la Cuenca Sinú: Reporte interno a la ANH con 2 tomos y 6 anexos, disponible en $<\mathrm{http}$ ://www.anh.gov. co/es/index.php?id=133> consultado 20 de junio de 2011 .

Woodring, W.P., 1973, Geology and Paleontology of Canal Zone and Adjoining Parts of Panama, Description of Tertiary Mollusks (Additions to gastropods, scaphopods, pelecypods: Nuculidae to Malleidae): Geological Survey, Professional paper, 306-E, 124 p.

Woods, M.A., 1922, Mollusca from the Eocene and Miocene deposits of Peru, en Bosworth, T.O. (ed), Geology of the Tertiay and Quaternary periods in the North-West part of Peru: MacMillan and Co. Ltd, London. 51-111.

Manuscrito recibido: Julio 28, 2015.

Manuscrito corregido recibido: Agosto 21, 2015.

Manuscrito aceptado: Octubre 22, 2015. 